\title{
A Computer Program for 3D Simulation of a Li-ion Half-Cell during Charging
}

Hamed Hosseinzadeh ${ }^{*}, 1$

${ }^{1}$ College of Engineering, Rowan University, Glassboro, New Jersey 08028, United States

Corresponding author: * College of Engineering, Rowan University, Glassboro, New Jersey 08028, United States Corresponding author email address: Hamed@uwalumni.com

Corresponding author Tel: + 18562835244 


\begin{abstract}
.
This code provides computational facilities to simulate current versus time during the charging of Li-ion cells at desire constant voltage by considering multiscale physical phenomena. This code only considers a powder of active materials (at microscale or nanoscale) and a small part of electrolyte around it as a half cell. Then it is extended to a complete cell by applying correct boundary conditions. This code is very useful by modifying code parameters to understand the effect of the complex shape of active materials powder (surface area and powder size), kind of electrolyte, and the applied voltages on the charging response of Li-ion cell. As a summary, a microscale approach to the design of Li-ion cells has been provided via this code.
\end{abstract}

\title{
Keywords:
}

Li-ion battery; Computer simulation; Numerical method; software

\section{Motivation and Significance}

Li-ion batteries are one of the most important technologies for energy storage applications and have applications for technologies like electric vehicles (EV) [1,2]. There are many computational and experimental studies on developing the performance of Li-ion battery and understanding underlying mechanisms [3-8]. Computer simulation and well-developed algorithms to perform a comprehensive simulation have highly demanded to design a more efficient Li-ion battery. Simulation of physical and chemical phenomena of Li-ion battery during the charging/discharging need a multiscale/multiphysics computational algorithms. As an instance, exerting a constant voltage on Li-ion cell to charge it would force the Li ions to move from cathode to anode, and this would be an atomic scale phenomenon. Meanwhile, the temperature and stress levels would change, which have an effect on Li-ion diffusion as well. Hence, an atomic scale phenomenon would be affected by two macroscale phenomena at different physics. There are several published papers on the simulation of intercalation induced stress [9], thermal behaviour [10], coupled thermal and stress evolution [11], electrochemical response and intercalation kinetics (at mesoscale [12-16] and meso-macroscale [17]) of Li-ion battery during charging and discharging. These published computer simulations have just explained mathematical aspects of governing equations, and they are not explaining their used codes and their structure. Besides thermal and intercalation stress, Li ions (de)intercalation in active materials is the primary step in developing code for simulating the electrochemical response of Li-ion cell. Then thermal/stress algorithm could be easily coupled to the intercalation kinetic algorithm in the code.

In this paper, a 3D code was developed to simulate the intercalation kinetic and electrochemical response of a half cell at the microscale. This code could be easily used for the whole cell, discharging, and larger scale. The overall algorithm, details of code, and outputs are explained in the following context.

\section{Software Description}

This article would explain code and its details to simulation Li-ions concentration (in the electrolyte and active material), potential (in the electrolyte and active material), and current versus time of Li-ion half-cell during charging. This code has been designed to charge a cell at a constant voltage by using multiscale/multiphysics algorithms. The computational scale of this code for electrochemical response and ionic diffusion is at the microscale. This code is also using a macroscale approach for simulating potential distribution in the electrolyte. In addition, another average method at the atomic scale is using to capture ion exchange at the interface of active materials and electrolyte, which is stochastic based on thermodynamic Li-ions activities in cathode and electrolyte. This part of code could be easily replaced by other methods like Butler-Volmer Equation. This code is simultaneously using these algorithms to simulate the overall response of Li-ion cells during charging. 


\subsection{Software Architecture}

The code has three coupled computational algorithms for Li-ion diffusion in active materials (here is a particle of the cathode with microscale size), Li-ion diffusion in the electrolyte, and an algorithm for making a decision on how Li-ions cross across the interface between active materials and electrolyte. The overall algorithm of this code has been shown in figure 1.

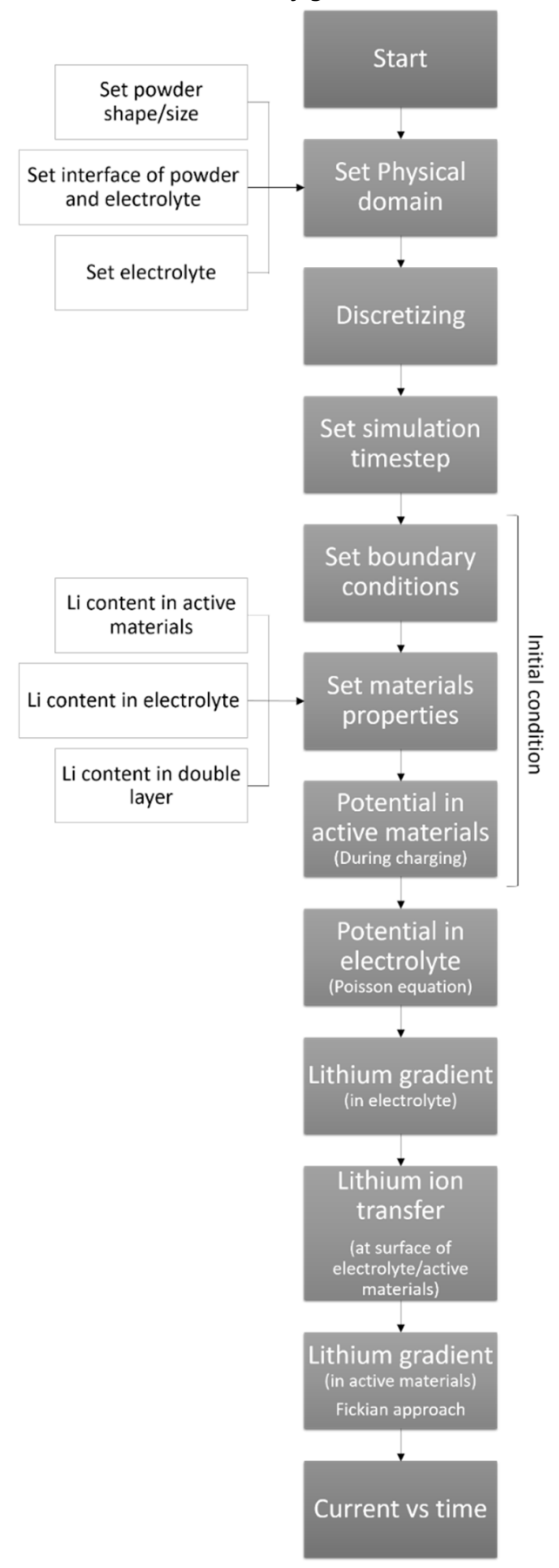

Figure 1. Used algorithm for simulating the electrochemical response of Li-ion cell. 


\subsection{Software Functionalities}

This code has a graphical user interface (GUI), which is written in Microsoft Visual basic. Figure 2 shows the GUI of this code, which is facilitating the simulation.

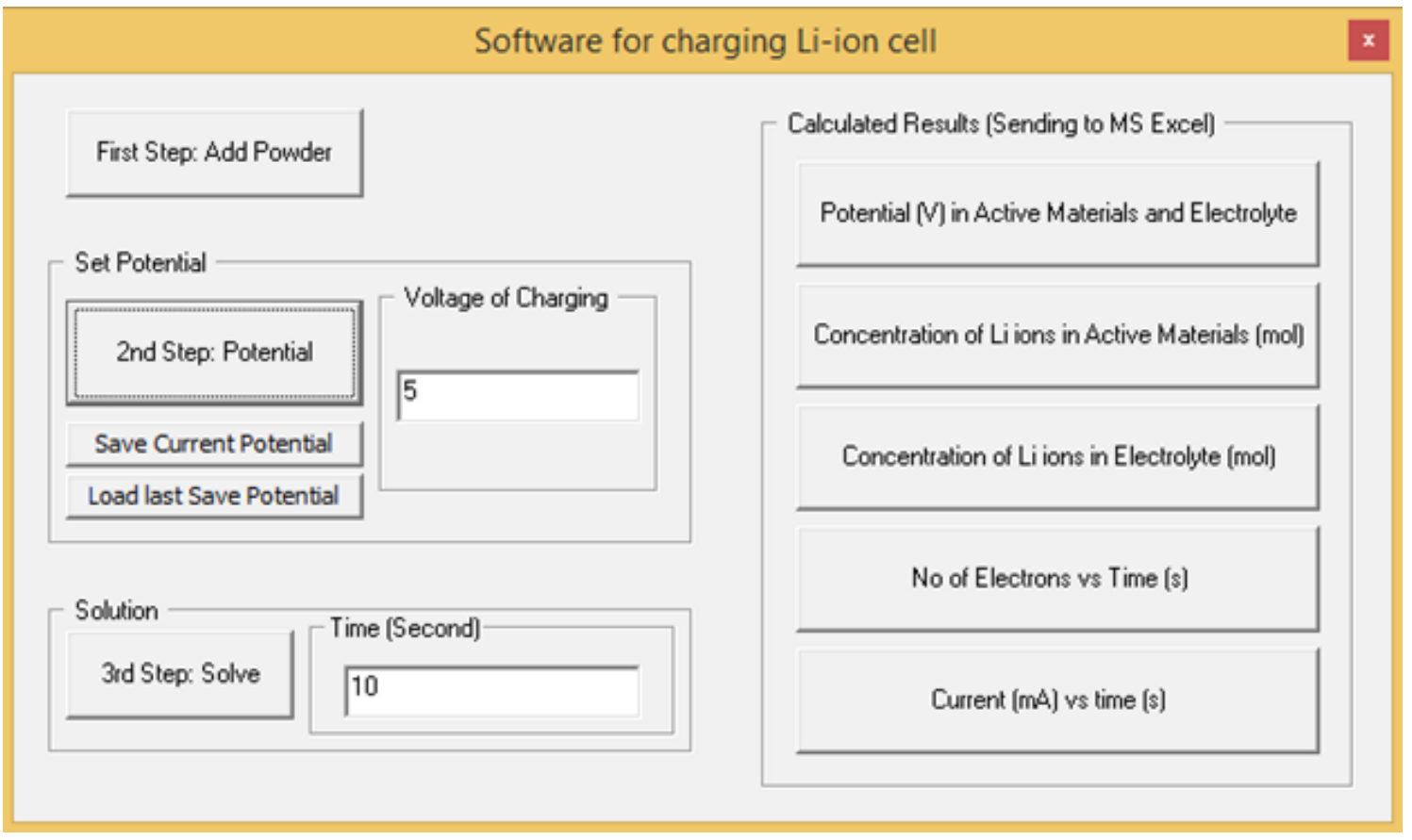

Figure 2. The graphical user interface of the code. It has two main portions. The first portion is for setting the physical environment and performing simulation; the second one is for retrieving calculated results (sending them to MS Excel).

The code is solving a conventional Fickian equation of ionic diffusion (Equation 1) with finite difference numerical method (FDM) for diffusion in active materials.

$$
j=-D \nabla C ; \frac{\partial C}{\partial t}=-\nabla \cdot j \rightarrow \frac{\partial C}{\partial t}=\nabla \cdot(D \nabla C)
$$

In equation $1, j$ is ionic flux, $D$ is ionic diffusion constant, $C$ is ionic concentration, and $t$ is time. This Fickian approach is also used for ionic diffusion in the electrolyte with regarding the effect of the local electrostatic potential on ionic diffusion, which is famous as the Nernst-Planck Equation (Equation 2).

$$
j=-D \nabla C-\frac{F}{R T} D z C \nabla \varphi ; \frac{\partial C}{\partial t}=-\nabla \cdot j \rightarrow \frac{\partial C}{\partial t}=\nabla \cdot\left(D \nabla C+\frac{F}{R T} D z C \nabla \varphi\right)
$$

In equation 2, $F$ is Faraday constant, $R$ is the universal gas constant, $T$ is temperature, $z$ is, and $\varphi$ is local electrostatic potential. Another algorithm is for solving the Poisson Equation (Equation 3) to calculate local electrostatic potential in the electrolyte, which is a necessary input in equation 2. This is an iterative solution method until acquiring relevant accuracy. 


$$
\frac{\partial^{2} \varphi}{\partial x^{2}}+\frac{\partial^{2} \varphi}{\partial y^{2}}+\frac{\partial^{2} \varphi}{\partial z^{2}}=\frac{\rho_{o}}{\varepsilon \varepsilon_{o}}
$$

In equation 3, $\rho_{o}$ is charge distribution, $\varepsilon$ is the relative permittivity of solvent and $\varepsilon_{o}$ is permittivity of free space. Applying external potential for charging electrochemical cells would introduce charges at the surface of active material, which is called the "electric double layer." In this case, active materials surface would positively charge, and ions with negative/positive charge would attract/repel to/from the active material surface during charging. In this case, an ion with a negative charge is $\mathrm{ClO}_{4}^{-}$and ion with a positive charge is $\mathrm{Li}^{+}$. Hence in this code, $\mathrm{Li}$ ions concentration sets/assume to zero in the electrolyte at several nanometres from the surface of active material to define the electrical double layer. This approach could be revised by any other more sophisticated ones in the code.

As mentioned before, the used numerical method is a finite difference method (FDM). Figure 3 shows the nodes and meshes configuration of FDM in this code.

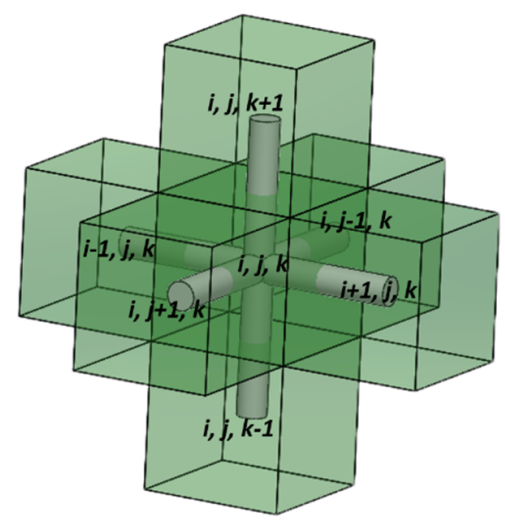

Figure 3. FDM meshes and their connections/notations.

Nodes are at the center of each cube, and the calculated result on each node would be extended to its cube. Hence, each cube is an FDM mesh in this code, and the center of it is a node.

Each node in this code transfers four data: 1. Spatial position, 2. Electrical potential, 3. Li-ions concentration, and 4. Kind of it (Active material node or electrolyte node or surface node). Before the solution, this code is specifying nodes of active materials, electrolytes and those at the surface of active materials/electrolytes. As mentioned before, each kind of these nodes has its own mathematical concept and equation. This subject has been shown in figure 4. 
(a)

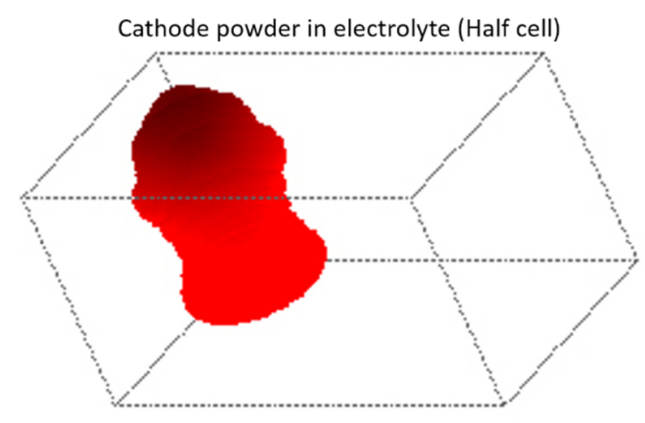

All elements at the surface of active

b) material powder

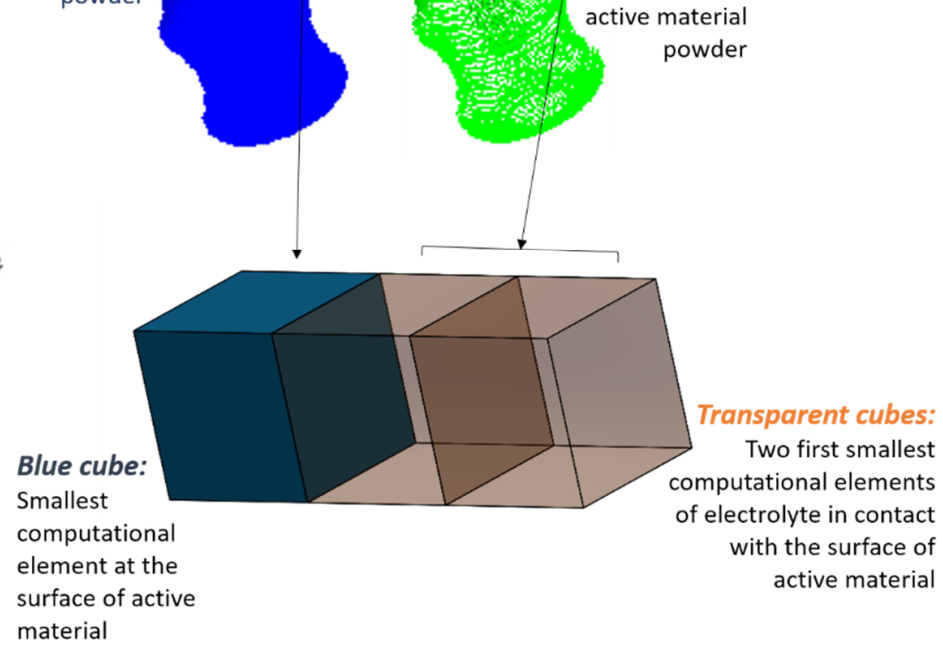

Figure 4. Before starting a solution, the kind of each node must be specified. (a) it shows a particle of the cathode in an electrolyte, (b) then software would identify nodes at the surface of cathode particle (blue ones) and their adjacent electrolyte nodes (green one). Ion exchange algorithm would apply to blue nodes, and the electrical double layer would define in green nodes.

It could simply translate equations 1 to 3 into computer code after FDM discretizing and identifying kind of each node. As an example, FDM discretized form of Poisson Equation (Equation 4) would be as follow:

$$
\begin{gathered}
\frac{\varphi_{i-1, j, k}-2 \varphi_{i, j, k}+\varphi_{i+1, j, k}}{\Delta x^{2}}+\frac{\varphi_{i, j-1, k}-2 \varphi_{i, j, k}+\varphi_{i, j+1, k}}{\Delta y^{2}}+\frac{\varphi_{i, j, k-1}-2 \varphi_{i, j, k}+\varphi_{i, j, k+1}}{\Delta z^{2}} \\
=\frac{\rho_{o}}{\varepsilon \varepsilon_{o}}
\end{gathered}
$$

Then the code of Equation 4 is as follow:

Dim P as Single 'Description: $P$ is variable for potential

Dim P_new as single 'Description: $P_{\text {_ }}$ new is variable for potential

Dim $d x$ as Single 'Description: $d x$ is numerical FDM mesh size

Start:

For $i, j$ and $k$ "for whole physical domain."

$$
\begin{aligned}
P \_ \text {new }(i, j, k)= & (P(i-1, j, k)+P(i+1, j, k)+P(i, j-1, k)+P(i, j+1, k)+P(i, j, k+1) \\
& +P(i, j, k-1)) / 6+\text { Constant number } * d x^{\wedge} 2
\end{aligned}
$$

Next

If $\mid P_{-}$new-P $\mid>$accuracy

For $i, j$ and $k$ "for whole physical domain"

$P(i, j, k)=P \_n e w(i, j, k)$

Next

Goto Start 


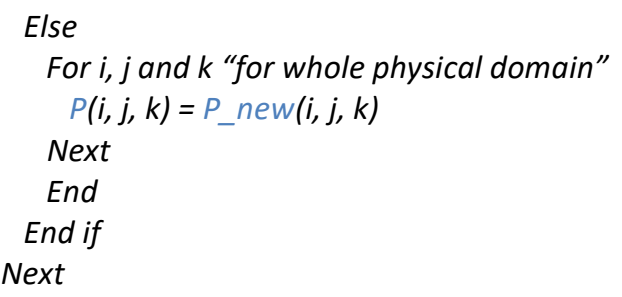

And code for the numerical solution of equation 1 is as follows:

Dim C as Single 'Description: P is variable for Li-ion concentration

Dim C_new as single 'Description: P_new is variable for Li-ion concentration at next time step

Dim $d x$ as Single 'Description: $d x$ is numerical FDM mesh size

Dim $d t$ as Single 'Description: $d t$ is the simulation time step

For $i, j$ and $k$ "for the whole physical domain."

$$
\begin{aligned}
& C_{\text {new }(i, j, k)}=C(i, j, k)+\left(D * d t / d x^{\wedge} 2\right) \\
& \\
& *(C(i-1, j, k)+C(i+1, j, k)+C(i, j-1, k)+C(i, j+1, k)+C(i, j, k+1) \\
&+C(i, j, k-1)-6 * C(i, j, k))
\end{aligned}
$$

Next

The FDM numerical stability condition must be satisfied to have a converged solution. In this case, Von Neumann stability condition (D. $\left.\Delta t / \Delta x^{2} \leq 0.5\right)$ is used for the diffusion equation in active materials and electrolyte. This code is using uniform mesh size; that is, mesh size of electrolyte and cathode nodes are the same. Li-ion diffusion constant of electrolyte is different from the cathode, which means the time step of ionic diffusion in the electrolyte is different from the cathode, according to Von Neumann's stability condition. So, the code runs one step of ionic diffusion in cathode material and then $K=$ $\Delta t_{\text {cathode }} / \Delta t_{\text {Electrolyte }}$ steps of ionic diffusion in the electrolyte.

The maximum numbers of nodes that could be set by the code are 2 million, and $\Delta t$ and $K$ must be then defined based on stability condition according to mesh size and ionic diffusion coefficient. As an example, the code was used to run a simulation. The simulation domain has shown in figure 5 (Although there is no $3 D$ visualization, this code exactly sets what in figure 5 as the simulation domain).

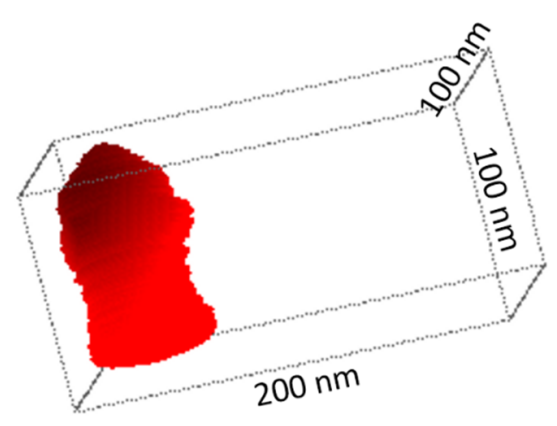

Figure 5. Simulation domain. Cathode particle (red) has a $20 \mathrm{~nm}$ average diameter and $90 \mathrm{~nm}$ height. The rest is an electrolyte. 
Li-ion diffusion coefficient in cathode and electrolyte were considered $10^{-14} \mathrm{~cm}^{2} \cdot \mathrm{s}^{-1}$ and $10^{-11} \mathrm{~cm}^{2} \cdot \mathrm{s}^{-1}$, respectively. The timestep for ionic diffusion in cathode was set 0.2 seconds, and for electrolyte was set $10^{-6}$ seconds. For this case, $K$ would be 200000 , but for simplicity, $K$ was set 2 in this simulation. Calculated results were shown in figure 6 (this half-cell was charged by five voltages for 400 seconds).
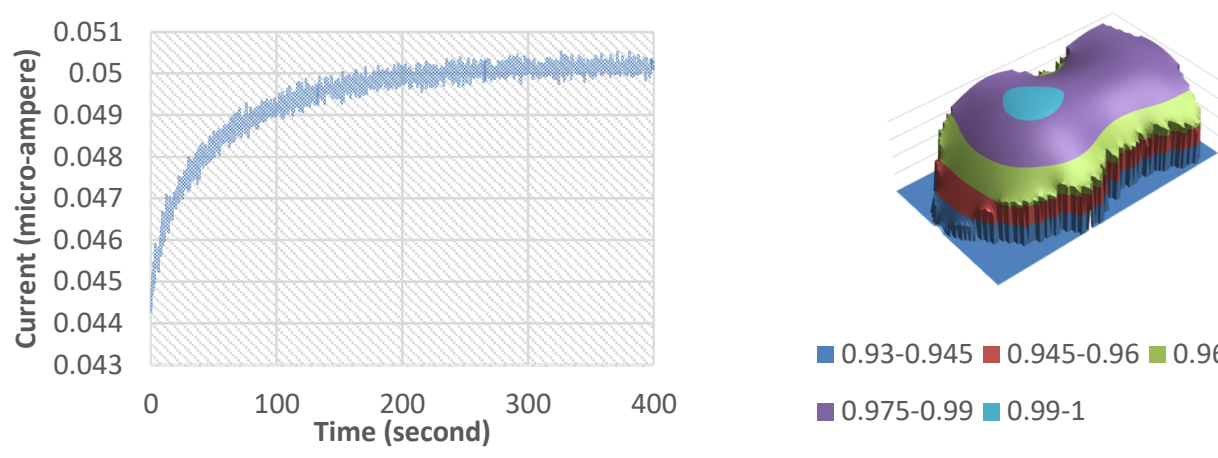

- 0.93-0.945 0.945-0.96 $=0.96-0.975$ 0.975-0.99 0.99-1

Figure 6. (a) Calculated current during charging with five constant voltage and (b) Li-ion concentration in the cathode particle after charging with five constant voltage for 400 seconds. Before charring, Li-ion concentration was 1 in cathode particle.

\section{Conclusions}

This is a code of 3-dimensional numerical simulation of the intercalation kinetic and electrochemical response for a Li-ion half-cell at the microscale. This code could be easily extended to any other applications, i.e., wholecell simulation, discharging as well, and larger scale by a slight change in algorithms because of the same concept of charging and discharging or the half-cell and complete cell. The code is freely available on a request from the author.

\section{References}

[1] L. Lu, X. Han, J. Li, J. Hua, M. Ouyang, J. Power Sources 226 (2013) 272-288.

[2] J.T. Warner, The Handbook of Lithium-Ion Battery Pack Design: Chemistry, Components, Types and Terminology, 2015.

[3] S.K. Mohammadian, Y. Zhang, J. Power Sources 273 (2015) 431-439.

[4] W. Wei, W. Lv, M.B. Wu, F.Y. Su, Y.B. He, B. Li, F. Kang, Q.H. Yang, Carbon N. Y. 57 (2013) 530-533.

[5] Y. Xie, J. Li, C. Yuan, J. Power Sources 248 (2014) 172-179.

[6] R. Zhao, J. Gu, J. Liu, J. Power Sources 266 (2014) 422-432.

[7] X. Sun, Y. Xu, P. Ding, M. Jia, G. Ceder, J. Power Sources 244 (2013) 690-694.

[8] I. Matts, H. Chen, G. Ceder, ECS Electrochem. Lett. 2 (2013) A81-A83.

[9] X. Zhang, W. Shyy, A. Marie Sastry, J. Electrochem. Soc. 154 (2007) A910.

[10] K. Onda, T. Ohshima, M. Nakayama, K. Fukuda, T. Araki, J. Power Sources 158 (2006) 535-542.

[11] X. Zhang, A.M. Sastry, W. Shyy, J. Electrochem. Soc. 155 (2008) A542.

[12] T. Hutzenlaub, S. Thiele, N. Paust, R. Spotnitz, R. Zengerle, C. Walchshofer, Electrochim. Acta 115 (2014) 131-139.

[13] G.B. Less, J.H. Seo, S. Han, A.M. Sastry, J. Zausch, A. Latz, S. Schmidt, C. Wieser, D. Kehrwald, S. Fell, J. Electrochem. Soc. 159 (2012) A697.

[14] C.-W. Wang, A.M. Sastry, J. Electrochem. Soc. 154 (2007) A1035.

[15] H. Seyyedhosseinzadeh, F. Mahboubi, A. Azadmehr, Electrochim. Acta 108 (2013) 867-875.

[16] H. Seyyedhosseinzadeh, F. Mahboubi, A. Azadmehr, lonics (Kiel). 21 (2014) 335-344. 
[17] S. Allu, S. Kalnaus, S. Simunovic, J. Nanda, J.A. Turner, S. Pannala, J. Power Sources 325 (2016) 42-50. 